K. D. Johnson

Nagoya Math. J.

Vol. 64 (1976), 17-29

\title{
DIFFERENTIAL EQUATIONS AND AN ANALOG OF THE PALEY-WIENER THEOREM FOR LINEAR SEMISIMPLE LIE GROUPS
}

\author{
KENNETH D. JOHNSON
}

\section{§1. Introduction}

Let $G$ be a noncompact linear semisimple Lie group. Fix $G=K A N$ an Iwasawa decomposition of $\mathrm{G}$. That is, $K$ is a maximal compact subgroup of $G, A$ is a vector subgroup with $A d A$ consisting of semisimple transformations and $A$ normalizes $N$, a simply connected nilpotent subgroup of $G$. Let $M^{\prime}$ denote the normalizer of $A$ in $K, M$ the centralizer of $A$ in $K$, and $W=M^{\prime} / M$ the restricted Weyl group of $G$. Fix $\theta$ a Cartan involution of $G$ which leaves every element of $K$ fixed and set $\bar{N}=\theta N$. We denote the Lie algebras of $G, K, A, N, \bar{N}$, and $M$ respectively by $\mathbb{S}, \mathfrak{R}, \mathfrak{A}, \mathfrak{\Re}, \overline{\mathfrak{N}}$, and $\mathfrak{M}$ respectively.

For $g \in G$ set $g=K(g) \exp H(g) n(g)$ where $K(g) \in K, H(g) \in \mathfrak{A}$, and $n(g) \in N$ and $\left.\exp \right|_{\mathfrak{r}}$ is an isomorphism from $\mathfrak{A}$ to $A$ with inverse $\log$. Recall that $\lambda \in \mathfrak{U}^{*}$ is called a root if $\mathscr{S S}_{\lambda}=\{X \in \mathscr{S}:[H, X]=\lambda(H) X$ for all $H \in \mathfrak{Q}\} \neq\{0\}$ and $\lambda$ is a positive root if $\mathscr{S}_{2} \subseteq \mathfrak{R}$. Let $P$ denote the set of all positive roots and let $L$ be the semilattice of all elements of $\mathfrak{U}^{*}$ of the form $\sum_{\lambda \in P} c_{\lambda} \lambda$ and $c_{\lambda}$ is a nonnegative integer.

Let $V$ be a finite dimensional vector space and let $K$ act on $V$ via the double representation $\tau$. That is, for $v \in V$ and $k_{1}, k_{2} \in K$

$$
\tau\left(k_{1}, k_{2}\right): v \longrightarrow \tau\left(k_{1}\right) \cdot v \cdot \tau\left(k_{2}\right)^{-1}
$$

Consider the $C^{\infty}$ functions $f: G \rightarrow V$ for which $f\left(k_{1} g k_{2}\right)=\tau\left(k_{1}\right) f(g) \tau\left(k_{2}\right)$ $\left(k_{1}, k_{2} \in K\right)$. We denote these functions by $C^{\infty}(G, \tau)$ and we denote the $C^{\infty}$-functions with compact support by $C_{c}^{\infty}(G, \tau)$ and the Schwartz functions in $C^{\infty}(G, \tau)$ by $\mathscr{C}(G, \tau)$.

Consider $f \in \mathscr{C}(G, \tau)$ and for $\nu \in \mathfrak{Y}_{\boldsymbol{C}}^{*} m \in M$ set

Received June 16, 1975. 


$$
g_{f}(\nu)(m)=\int_{A} d a \int_{N} f(\operatorname{man}) e^{(\rho-i \nu)(\log a)} d n
$$

where for $H \in \mathfrak{A} \rho(H)=\frac{1}{2} \operatorname{tr} a d H_{\mid \Re}$ and for $\omega \in \hat{M}$, set

$$
\psi_{f}(\omega: \nu)=\int_{M} \chi_{\omega}\left(m^{\prime}\right) g_{f}(\nu)\left(m^{\prime}\right) d m^{\prime}
$$

Now $\psi_{f}(\omega: \nu) \in V^{M}$ where $V^{M}=\{v \in V: \tau(m) v=v \tau(m)$ for all $m \in M\}$ and in fact $\psi_{f}(\omega: \nu) \in V^{M}(\omega)$ where $V^{M}(\omega)=E_{\omega}\left(V^{M}\right)$ and

$$
E_{\omega}(v)=d_{\omega} \int_{M} \overline{\chi_{\omega}(m)} \tau(m) v d m
$$

In general for $A \in V^{M}$ we define the Eisenstein integral of HarishChandra by setting

$$
E(A: \nu: x)=\int_{K} \tau(K(x k)) \circ A \circ \tau(k)^{-1} e^{(i \nu-\rho)(H(x k))} d k .
$$

Remark. Our notation for the Eisenstein integral differs slightly from Harish-Chandra's Eisenstein integral only in that we shall have no need to specify the parabolic subgroup $P=M A N$ which defines the integral.

Part of the Plancherel formula of Harish-Chandra [6], [7] tells us that for $f \in \mathscr{C}(G, \tau)$ there is a function $f_{A} \in \mathscr{C}(G, \tau)$ where

$$
f_{A}(x)=\sum_{\omega \in \hat{M}} \int_{\mathfrak{x}^{*}} E\left(\psi_{f}(\omega: \nu): \nu: x\right) \mu(\omega: \nu) d \nu
$$

and $F=f-f_{A} \in \mathscr{C}(G, \tau)$ with

$$
\int_{N} F(g n) d n \equiv 0
$$

where $N$ is the unipotent radical of $P=M A N$. Moreover, the function $\mu: \hat{M} \times \mathfrak{Y}_{C}^{*} \rightarrow C$ satisfies the following conditions:

1) $\nu \rightarrow \mu(\omega: \nu)$ is meromorphic on $\mathfrak{X}_{C}^{*}(\omega \in \hat{M})$;

2) $\nu \rightarrow \mu(\omega: \nu)$ is analytic and $\geq 0$ on $\mathfrak{X}^{*}(\omega \in \hat{M})$; and,

3) For $s \in W \mu(s \omega: s \nu)=\mu(\omega: \nu)$.

In the following we will say that a function $F \in \mathscr{C}(G, \tau)$ is a quasicusp form if

$$
\int_{N} F(g n) d n \equiv 0
$$


We denote the space of quasi-cusp forms by $\mathscr{C}_{q}(G, \tau)$.

The main result of this paper (Theorem 3.1) gives a weak analog of the classical Paley-Wiener theorem in characterizing the support of a function $f \in C_{c}^{\infty}(G, \tau)$ in terms of growth conditions on the "FourierLaplace transform" $\psi_{f}(\omega: \nu)$.

We first state some results concerning some estimates which we shall need in the proof of the Paley-Wiener theorem.

In Section 3 we prove our result which contains a rather ambiguous residue function which we treat somewhat further in Section 4 . In Section 5 we apply our results to the study of some partial differential operators on $G$.

\section{§2. Some estimates.}

Let $V$ be as in section one, let $A \in V^{M}$ and consider the Eisenstein integral $E(A: \nu: x)$. Let $\mathfrak{U}^{+}=\{H \in \mathfrak{U}: \lambda(H)>0$ for all $\lambda \in P\}$ and set $A^{+}$ $=\exp \mathfrak{U}^{+}$. Harish-Chandra in Warner [16] has given a useful expansion of $E(A: \nu: a)$ for $a \in A^{+}$which we now describe.

For $a \in A^{+}$and $s \in W$ there exist functions $c: W \times \mathfrak{A}_{C}^{*} \rightarrow$ End $V^{M}$ and $\Phi_{s}: A \times \mathfrak{U}_{C}^{*} \rightarrow$ End $V^{M}$ such that $E(A: \nu: a)=\sum_{s \in W} \Phi_{s}(a: \nu)(c(s: \nu)(A))$. Furthermore, we have that

$$
\Phi_{s}(a: \nu)=\sum_{\mu \in L} \Gamma_{\mu}(i s \nu-\rho) e^{(i s \nu-\rho-\mu)(\log a)}
$$

where for $\mu \in L \nu \rightarrow \Gamma_{\mu}(i s \nu-\rho)$ is a rational function with image in End $\left(V^{M}\right)$. Here $\Gamma_{0}=I$.

For $\lambda \in \mathfrak{Y} *$ there is an $H_{\lambda} \in \mathfrak{X}$ such that $\lambda(H)=B\left(H, H_{\lambda}\right)$ for all $H \in \mathfrak{A}$ where $B$ is the Killing form of $\mathbb{s}$. For $\nu \in \mathfrak{X}_{c}^{*}$ write $-i \nu=\xi+i \eta$ when $\xi, \eta \in \mathfrak{Y}^{*}$. For $H_{0} \in \mathfrak{X}$ set $T\left(H_{0}\right)=\left\{\nu \in \mathfrak{U}_{c}^{*}: H_{\xi} \in H_{0}+\mathfrak{U}^{+}\right\}$. The $\Gamma_{\mu}$ 's now satisfy the following

Lemma 2.1 (Lemma 2.3 [13]). Fix $H_{0} \in \mathfrak{A}$ and $H_{1} \in \mathfrak{U}^{+}$. Then there is a polynomial $p_{H_{0}}(\nu)$ and a polynomial $K(\nu)>0$ depending on $p_{H_{0}}, H_{0}$ and $H_{1}$ such that

$$
\left\|p_{H_{0}}(\nu) \Gamma_{\mu}(i \nu-\rho)\right\| \leq K e^{\mu\left(H_{1}\right)} .
$$

For the proof of this lemma we refer to [13]. We now need some estimates on the functions $c(s: \nu)$.

We say that for $a \in A^{+} a \rightarrow \infty$ if $\|\log a\|=B(\log a, \log a)^{1 / 2} \rightarrow \infty$ and 
there is an $\varepsilon>0$ such that for all $\lambda \in P \lambda(\log a) \geq \varepsilon\|\log a\|$. Then from Harish-Chandra [6], [7] we have for $A \in V^{M}$ and $\nu \in \mathfrak{X}^{*}$ that

$$
\lim _{a \rightarrow \infty}\left(e^{\rho(\log a)} E(A: \nu: a)-\sum_{s \in W} c(s: \nu)(A) e^{i s \nu(\log a)}\right)=0 .
$$

Again from Harish-Chandra [6], [7] we have that the map $\nu \rightarrow c(s: \nu) \in$ End $\left(V^{M}\right)$ is meromorphic and hence we see that if $\operatorname{Re} i \nu(\log a)>0$ for all $a \in A^{+}$

$$
\log _{a \rightarrow \infty} e^{(\rho-i \nu)(\log a)} E(A: \nu: a)=c(1: \nu)(A) .
$$

Hence for $\operatorname{Re} i \nu(\log a)>0$ and all $a \in A^{+}$we obtain

$$
c(1: \nu)=\int_{\bar{N}} A \circ \tau(K(\bar{n}))^{-1} e^{-(i \nu+\rho)(H(\pi))} d \bar{n} .
$$

More generally we obtain that if $\operatorname{Re} i s \nu(\log a)>0$ for all $a \in A^{+}$and $s \in W$

$$
\log _{a \rightarrow \infty} e^{(\rho-i s \nu)(\log a)} E(A: \nu: a)=c(s: \nu)(A)
$$

and in this case an elementary calculation yields

$$
c(s: \nu)(A)=\tau(w) j_{s}^{-}(\nu) \circ A \circ j_{s}^{+}(\nu) \tau(w)^{-1} \quad(w \in s)
$$

where

$$
j_{s}^{+}(\nu)=\int_{\bar{N}_{1}} e^{-(i \nu+\rho) H(\tilde{n})} \tau(K(\bar{n}))^{-1} d \bar{n}
$$

and

$$
j_{s}^{-}(\nu)=\int_{\bar{N}_{2}} e^{(i \nu-\rho) H(\pi)} \tau(K(\bar{n})) d \bar{n}
$$

with $\bar{N}_{1}=\left\{\bar{n} \in \bar{N}: w \bar{n} w^{-1} \in \bar{N}\right\}$ and $\bar{N}_{2}=\left\{\bar{n} \in \bar{N}: w \bar{n} w^{-1} \in N\right\}$.

We wish to apply estimates of the form found in Lemma 3.1 of [13]. To do so we first need a product formula for the functions $j_{s}^{+}(\nu)$ and $j_{s}^{-}(\nu)$ which may be attributed to Gindikin and Karpelevic [4] and Schiffmann [15]. A more general product formula has been obtained by Harish-Chandra [7].

Let $P_{s}^{+}=\left\{\alpha \in P: s^{-1} \alpha>0\right\}$ and $P_{s}^{-}=\left\{\alpha \in P: s^{-1} \alpha<0\right\}$. Then

$$
\overline{\mathfrak{N}}_{1}=\sum_{\alpha \in P_{s}^{+}} \mathbb{S}_{-\alpha} \text { and } \overline{\mathfrak{R}}_{2}=\sum_{\alpha \in P_{\bar{s}}^{-}} \mathscr{S}_{-\alpha}
$$

and for $\alpha \in P$ where $\alpha / 2 \in P$ let $\mathfrak{N}_{\alpha}=\mathbb{S}_{-\alpha}+\mathbb{S}_{-2 \alpha}$. If $\alpha \in P_{s}^{+}$set 


$$
j_{\alpha}^{+}(\nu)=\int_{\bar{N}_{\alpha}} e^{-(i \nu+\rho)(H(\tilde{n}))} \tau(K(\bar{n}))^{-1} d \bar{n}
$$

and if $\alpha \in P_{s}^{-}$set

$$
j_{\alpha}^{-}(\nu)=\int_{\bar{N}_{\alpha}} e^{\left(i_{\nu}-\rho\right) H(\bar{n})} \tau(K(\bar{n})) d \bar{n}
$$

If $\left|P_{s}^{+}\right|=k$ and $\left|P_{s}^{-}\right|=\ell$ we may put an ordering on $P_{s}^{+}$where $P_{s}^{+}=$ $\left\{\alpha_{1}, \cdots, \alpha_{k}\right\}$ on an ordering on $P_{s}^{-}$where $P_{s}^{-}=\left\{\lambda_{1}, \cdots, \lambda_{\ell}\right\}$ where $\alpha_{i} \leq \alpha_{i+1}$ and $\lambda_{i} \leq \lambda_{i+1}$ such that $j_{s}^{+}(\nu)=j_{\alpha_{k}}^{+}(\nu) \cdots j_{\alpha_{1}}^{+}(\nu)$ and $j_{s}^{-}(\nu)=j_{\lambda_{l}}^{-}(\nu) \cdots j_{\lambda_{1}}^{-}(\nu)$. The proof of this fact follows immediately from Gindikin-Karpelevic [4] or more precisely from the proof of their main theorem. From Lemma 3.2 of [13] we have the following lemma

LEMMA 2.2. Given $\delta>0$ there is an $R>0$ and an integer $N>0$ such that if $|\langle\nu, \alpha\rangle|>R$ and $|\arg \langle\nu, \alpha\rangle+\pi / 2| \geq \delta$ for $\alpha \in P_{s}^{+}$the matrix entries of $j_{\alpha}^{+}(\nu)^{-1}$ are bounded in absolute value by $|\langle\nu, \alpha\rangle|^{N}$. Hence there is an $R_{1}>0$ and an $N_{1}>0$ for which the matrix entries of $j_{s}^{+}(\nu)^{-1}$ are bounded in absolute value by $\pi_{\alpha \in P_{s}^{+}}|\langle\nu, \alpha\rangle|^{N_{1}}$ if $\left.|\langle\nu, \alpha\rangle|\right\rangle R$, and $\mid \arg \langle\nu, \alpha\rangle$ $+\pi / 2 \mid \geq \delta$ for $\alpha \in P_{s}^{+}$. (Here $|\arg z| \leq \pi$.) Furthermore there is an $R^{\prime}>0$ and an integer $N^{\prime}>0$ for which the matrix entries of $j_{s}^{-(\nu)^{-1}}$ are bounded in absolute value by $\pi_{\alpha \in P_{s}^{-}}|\langle\nu, \alpha\rangle|^{N^{\prime}}$ if $\left.|\langle\nu, \alpha\rangle|\right\rangle R^{\prime}$ and $|\arg \langle\nu, \alpha\rangle-\pi / 2|$ $\geq \delta$ for $\alpha \in P_{s}^{-}$.

Using the inner product on $V^{M}$ we now compute the adjoint of $c(s: \nu)$ for $\nu \in \mathfrak{Y}^{*}$. Fixing $w \in s$ as before and letting $B \in$ End $V^{M}$, we see that

$$
c(s: \nu) *(B)=\left(j_{s}^{-}(\nu)\right)^{*} \tau(w)^{-1} B \cdot \tau(w)\left(j_{s}^{+}(\nu)\right)^{*} .
$$

Moreover, we see that $\left(j_{s}^{-}(\nu)\right)^{*}$ is the limit of operators of the form

$$
\int_{\vec{N}_{2}} e^{-(i \lambda+\rho) H(\pi)} \tau(K(\bar{n}))^{-1} d \bar{n}
$$

where $\lambda \rightarrow \nu\left(\nu \in \mathfrak{I}^{*}\right)$ and $\left(j_{s}^{+}(\nu)\right)^{*}$ is the limit of operators of the form

$$
\int_{\bar{N}_{\mathbf{1}}} e^{(i \lambda-\rho)(H(\bar{n}))} \tau(K(\bar{n})) d \bar{n}
$$

where $\lambda \rightarrow \nu\left(\nu \in \mathfrak{O}^{*}\right)$.

We now compute the adjoint of $c(s: \nu)$ for $\nu \in \mathfrak{Y}^{*}$. For $w \in s$ and $B \in V^{M}$ we see that 


$$
c(s: \nu)^{*}(B)=j_{s}^{-}(\nu)^{*} \circ \tau(w)^{-1} \circ B \circ \tau(w) \circ j_{s}^{+}(\nu)^{*} .
$$

For $\lambda \in \mathfrak{A}_{\boldsymbol{C}}^{*}$ let

$$
J_{s}^{-}(\lambda)=\int_{\bar{N}_{2}} e^{-(i \lambda+\rho)(H(\bar{n}))} \tau(K(\bar{n}))^{-1} d \bar{n}
$$

and

$$
J_{s}^{+}(\lambda)=\int_{\bar{N}_{1}} e^{(i \lambda-\rho)(H(\bar{n}))} \tau(K(\bar{n})) d \bar{n}
$$

and denote their meromorphic continuations by the same symbols. Then $\left(j_{s}^{-}(\nu)\right)^{*}=J_{s}^{-}(\nu)$ and $\left(j_{s}^{+}(\nu)\right)^{*}=J_{s}^{+}(\nu)$. Letting $\tilde{C}(s: \lambda)(B)=J_{s}^{-}(\lambda) \tau(w)^{-1} B \tau(w)$ $\times J_{s}^{+}(\lambda)$ we see that the function $\lambda \rightarrow \tilde{C}(s: \lambda)$ is defined meromorphically and for $\nu \in \mathfrak{A}^{*} \tilde{C}(s: v)=c(s: \nu)^{*}$. It is a trivial fact to see that $J_{s}^{-}(\nu)=$ $j_{\lambda_{1}}^{+}(\nu) \cdots j_{\lambda_{\ell}}^{+}(\nu)$ and $J_{s}^{+}(\nu)=j_{\alpha_{1}}^{-}(\nu) \cdots j_{\alpha_{k}}^{-}(\nu)$ where the $\alpha_{i}$ and $\lambda_{j}$ are as before.

We conclude this section with the following observation. Suppose $f$ is a holomorphic function on $C^{n}$ and suppose that $f$ satisfies the following estimate. There are constants $C$ and $A>0$ and an integer $N>n$ for which

$$
|f(\vec{z})| \leq C(1+\|\vec{z}\|)^{-N} e^{A|| \operatorname{Im} \vec{z} \|}
$$

where $\|\vec{z}\|=(\langle\vec{z}, \vec{z}\rangle)^{1 / 2}$ and for $\vec{z}=\vec{x}+i \vec{y}$ with $\vec{x}, \vec{y} \in \boldsymbol{R}^{n} \operatorname{Im} \vec{z}=\vec{y}$.

Suppose $m>0$ is an integer and let $c_{1}, \cdots, c_{n}, \lambda \in C$. We assume that $\left\{\vec{z}: c_{1} z_{1}+\cdots+c_{n} z_{n}-\lambda=0\right\} \cap \boldsymbol{R}^{n}=\emptyset$. Let $g(\vec{z})=(\vec{c} \cdot \vec{z}-\lambda)^{-m} f(\vec{z})$ where $\vec{c}=\left(c_{1}, \cdots, c_{n}\right)$. Then the following formula holds.

$$
\begin{aligned}
& \int_{-\infty}^{\infty} \cdots \int_{-\infty}^{\infty} g\left(x_{1}, \cdots, x_{n}\right) d x_{1} \cdots d x_{n} \\
&= \int_{-\infty}^{\infty} \cdots \int_{-\infty}^{\infty} g\left(x_{1}+i y, x_{1}, \cdots, x_{n}\right) d x_{1} \cdots d x_{n} \\
&-2 \pi i \int_{-\infty}^{\infty} \cdots \int_{-\infty}^{\infty} \operatorname{Res}_{z}\left(g\left(z, x_{2}, \cdots, x_{n}\right),\right. \\
&\left.\frac{\lambda-c_{2} x_{2}-\cdots-c_{n} x_{n}}{c_{1}}\right) d x_{2} \cdots d x_{n}
\end{aligned}
$$

The above observation is useful since the singularities of the function $\nu \rightarrow \Gamma_{\mu}(i s \nu-\rho)(\mu \in L)$ and $\nu \rightarrow c(s: \nu)^{-1}$ have their singularities on hyperplanes and are meromorphic with polynomial growth.

\section{§3. A Paley-Wiener theorem}

We now describe our analog of the classical Paley-Wiener theorem. 
We suppose first that $f \in C_{c}^{\infty}(G, \tau)$ and $f(g)=0$ for $\sigma(g)>A$ where if $g=k_{1} a k_{2}$ with $k_{1}, k_{2} \in K$ and $a \in A \sigma(g)=(B(\log a, \log a))^{1 / 2}$ or we say $f \in C_{A}^{\infty}(G, \tau)$. Observe that the map $\nu \rightarrow \psi_{f}(\omega: \nu)$ is holomorphic and satisfies

(1) For $N>0$ an integer there is a constant $C_{N}$ such that

$$
\left\|\psi_{f}(\omega: \nu)\right\| \leq C_{N}(1+\|\nu\|)^{-N} e^{A\|\operatorname{Im} \nu\|} .
$$

(2) For $s \in W$ we have

$$
c(s: \nu)\left(\psi_{f}(\omega: \nu)\right)=c(1: s \nu)\left(\psi_{f}(s \omega: s \nu)\right) .
$$

We now derive a third condition which is satisfied by the function $\nu \rightarrow \psi_{f}(\omega: \nu)$ for $\omega \subset \tau_{\mid M}$. We have that

$$
f_{A}(g)=\sum_{\omega \in \hat{M}} \int_{\mathscr{Q}^{*}} E\left(\psi_{f}(\omega: \nu): \nu: g\right) \mu(\omega: \nu) d \nu .
$$

Moreover, picking an $\eta \in \mathfrak{U}^{*}$ with $\|\eta\|$ small and with no $\nu \rightarrow \Gamma_{\mu}(i s(\nu+i \eta)$ $-\rho)(\mu \in L)$ having a singularity for any $\nu \in \mathfrak{Y}^{*}$ we have

$$
f_{A}(g)=\sum_{\omega \in \hat{M}} \int_{\mathscr{Q}^{*}} E\left(\psi_{f}(\omega: \nu+i \eta): \nu+i \eta: g\right) \mu(\omega: \nu+i \eta) d \nu
$$

and by Lemma 2.1 we have for $a \in A^{+}$

$$
f_{A}(a)=\sum_{s \in w} \sum_{\omega \in \hat{M}} \sum_{\mu \in L} \int_{\mathfrak{R}^{*}+i \eta} \Gamma_{\mu}(i s \nu-\rho) c(s: \nu)\left(\psi_{f}(\omega: \nu)\right) \mu(\omega: \nu) e^{(i s \nu-\rho-\mu)(\log a)} d \nu
$$

The Maass-Selberg relations of Harish-Chandra [6], [7] state that

$$
\left\|c(s: \nu)\left(\psi_{f}(\omega: \nu)\right)\right\|^{2}=\left\|\tilde{C}(s: \nu)\left(\psi_{f}(\omega: \nu)\right)\right\|^{2}=\mu(\omega: \nu)^{-1} d_{\omega}\left\|\psi_{f}(\omega: \nu)\right\|^{2}
$$

for $\nu \in \mathfrak{X} *$. Hence we have $\mu(\omega: \nu)^{-1} d_{\omega}=c(s: \nu) \tilde{C}(s: \nu)_{\mid V^{M}(\omega)}$. Thus,

$$
\mu(\omega: \nu) c(s: \nu)\left(\psi_{f}(\omega: \nu)\right)=d_{\omega} \tilde{C}(s: \nu)^{-1}\left(\psi_{f}(\omega: \nu)\right) .
$$

For $H \in \mathfrak{A}$ and $s \in W$ consider the tube $T(s, H)=\left\{\nu \in \mathfrak{A}_{c}^{*}:-H_{\operatorname{Im} s \nu} \in \mathfrak{U}^{+}\right.$ $+H\}$. Then the following lemma now follows from Lemmas 2.1 and 2.2

LEMma 3.1. Given $H_{\eta} \in \mathfrak{A}$ and $s \in W$ there are a finite number of hyperplanes $F_{1}, \ldots, F_{r}$ in $\mathfrak{H}_{c}^{*}$ which intersect $T\left(s, H_{\eta}\right)$ and for which the functions $\nu \rightarrow \Gamma_{\mu}(i s \nu-\rho)(\mu \in L)$ and $\nu \rightarrow \tilde{C}(s: \nu)^{-1}$ are analytic on $T(s, H)$ $\sim\left(F_{1} \cup \cdots \cup F_{r}\right)$. Furthermore, there is a $C>0$ such that $\{\nu:-\langle\operatorname{Im} \nu, \alpha\rangle$ $>C$ for all $\alpha \in P\} T(s, H) F_{i}=\emptyset$ for all $1 \leq i \leq r$.

Now setting for $\mathrm{s} \in W$ and $a \in A^{+}$, 


$$
f_{A, s}(a)=\sum_{\mu \in \hat{M}} \sum_{\mu \in L} d_{\omega} \int_{\mathfrak{Q}^{*}+i \eta} \Gamma_{\mu}(i s \nu-\rho) \tilde{C}(s: \nu)^{-1}\left(\psi_{f}(\omega: \nu)\right) d \nu
$$

Using our remarks at the end of Section 2, we see that $f_{A, s}(a)=$ $\operatorname{Res}_{s}(f)(a)+f_{s, s}(a)$ where $\operatorname{Res}_{s}(f)(a)$ is a residue integral over the imaginary part of the hyperplanes $F_{1}, \cdots, F_{r}$ and

$$
f_{\varepsilon, s}(a)=\sum_{\omega \in \hat{M}} \sum_{\mu \in L} \int_{\operatorname{Im} \nu=\lambda} \Gamma_{\mu}(i s \nu-\rho) \tilde{C}(s: \nu)^{-1}\left(\psi_{f}(\omega: \nu)\right) e^{(i s \nu-\rho-\mu)(\log a)} d \nu
$$

with $-H_{\lambda} \in \mathfrak{O}^{+}$and $\|\lambda\|>C$. By the standard method used in the classical Paley-Wiener theorem we see that $f_{s, s}(a)=0$ if $\sigma(a)>A$. Letting $\operatorname{Res}(f)=\sum_{s \in w} \operatorname{Res}_{s}(f)$ and $f_{s}=\sum_{s \in w} f_{s, s}$ and using the Plancherel formula we now see that there is an $F \in \mathscr{C}_{q}(G, \tau)$ such that

(3) $f=f_{s}+\operatorname{Res}(f)+F$

and $\operatorname{Res} f(a)+F(a)=0$ for $a \in A^{+}$with $\sigma(a)>A$.

Now for $A>0$ let $\mathscr{P}(A, \tau)$ be the space of all functions $F: \hat{M} \times \mathfrak{U}_{c}^{*}$ $\rightarrow V$ such that $F(\omega: \nu) \equiv 0$ if $\omega \succ \tau_{\mid M}$ and $F$ satisfies the following conditions.

I) $\nu_{N}(F)=\sup _{\omega, \nu}(1+\|\nu\|)^{N} e^{-A|\mathrm{Im} \nu|}\|F(\omega: \nu)\|<\infty$

II) $c(s: \nu)(F(\omega: \nu))=c(1: s \nu)(F(s \omega: s \nu))$

III) The function

$$
f(g)=\sum_{\omega \in \hat{M}} \int_{\mathfrak{r}^{*}} E(F(\omega: \nu): \nu: g) \mu(\omega: \nu) d \nu
$$

differs from a function in $C_{c}^{\infty}(G, \tau)$ by a function $H$ in $\mathscr{C}_{q}(G, \tau)$. Moreover, for $g$ regular $f(g)=\operatorname{Res} f(g)+f_{c}(g)$ with $f_{c}(g)=0$ for $\nabla(g)>A$.

THEOREM 3.1. A function $f \in C^{\infty}(G, \tau)$ is in $C_{A}^{\infty}(G, \tau)+\mathscr{C}_{q}(G, \tau)$ if and only if its Fourier-Laplace transform is in $\mathscr{P}(A, \tau)$.

Proof. It is clear that if $f \in C_{A}^{\infty}(G, \tau)+\mathscr{C}_{q}(G, \tau)$ its Fourier-Laplace transform is in $\mathscr{P}(A, \tau)$.

Suppose $0 \neq F \in \mathscr{P}(A, \tau)$. By Theorem 3.1 of Arthur [1] we have that

$$
f(g)=\sum_{\omega \in \hat{M}} \int_{\mathfrak{Q}^{*}} E(F(\omega: \nu): \nu: g) \quad \mu(\omega: \nu) d \nu \not \equiv 0 .
$$

By Lemma 2.2 of [13] we have that $f \notin \mathscr{C}_{q}(G, \tau)$. By assumption there is an $H \in \mathscr{C}_{q}(G, \tau)$ for which $f-H \in C_{c}^{\infty}(G, \tau)$. However our arguments in obtaining 3) guarantee that $0 \neq f-H \in C_{A}^{\infty}(G, \tau)$. This completes our proof. 
Corollary 1. A function $f \in C_{c}^{\infty}(G, \tau)$ is in $C_{A}^{\infty}(G, \tau)$ if and only if for every integer $N>0$ there is a $C_{N}>0$ such that

$$
\left\|\psi_{f}(\omega: \nu)\right\| \leq C_{N}(1+\|\nu\|)^{-N} e^{A\|\operatorname{Im} \nu\|} .
$$

CoRollary 2. Let $\mathscr{P}(\tau)$ be the union of all $\mathscr{P}(A, \tau)$. Then a function $f \in C^{\infty}(G, \tau)$ is in $C_{c}^{\infty}(G, \tau)+\mathscr{C}_{q}(G, \tau)$ if and only if its Four ier-Laplace transform is in $\mathscr{P}(\tau)$.

\section{§4. The function $\operatorname{Res} f$}

We inject here a few remarks concerning the function $\operatorname{Res} f$ where $f \in C_{A}^{\infty}(G, \tau)$. Although we have strong reason to believe that $\operatorname{Res} f$ extends to a function in $\mathscr{C}_{q}(G, \tau)$ and thus $f_{s}$ extends to a function in $C_{A}^{\infty}(G, \tau)$ we can only establish this for some special cases which we describe in this section. We first give a more detailed description of $\operatorname{Res} f$.

Let $P$ denote the set of positive restricted roots and let $\Delta=\left\{\alpha_{1}, \cdots\right.$, $\left.\alpha_{\ell}\right\}$ be the simple restricted roots in $P$. Let $\left\{\lambda_{1}, \cdots, \lambda_{\ell}\right\}=' \Delta$ be dual to $\Delta$ (i.e. $\left.2\left(\left\langle\lambda_{i}, \alpha_{j}\right\rangle /\left\langle\alpha_{j}, \alpha_{j}\right\rangle\right)=\delta_{i j}\right)$. For $F \subset \Delta$ let ${ }^{c} F=\Delta \sim F$ and let $F \subset \subset^{\prime} \Delta$ be dual to $F$ and ${ }^{\circ} F$ dual to ${ }^{c} F$. Let $\mathfrak{U}(F)\left(\mathscr{U}\left({ }^{\circ} F\right)\right)$ be the linear span of $\left\{H_{\alpha}: \alpha \in F\right\} \quad\left(\left\{H_{\alpha}: \alpha \in{ }^{\circ} F\right\}\right)$ and set $A(F)=\exp \mathfrak{Y}(F)\left(A\left({ }^{\circ} F\right)=\exp \mathfrak{Y}\left({ }^{\circ} F\right)\right)$. Observe that if $H \in \mathfrak{U} H=H_{1}+H_{2}$ where $H_{1} \in \mathfrak{U}(F)$ and $H_{2} \in \mathfrak{U}\left({ }^{\circ} F\right)$ and this decomposition is unique. Furthermore, if $H \in \mathfrak{U}^{+} H=H_{1}+H_{2}$ where $H_{1} \in \mathfrak{U}\left(F^{\prime}\right)^{+}=\{H \in \mathfrak{A}(F): \alpha(H)>0$ for all $\alpha \in F\}$ and $H_{2}=\sum c_{\lambda} H_{\lambda}$ where the sum is over ${ }^{\circ} F$ and each $c_{\lambda}>0$. (It is easy to see that the converse holds only when $F=\Delta$ or $F=\emptyset$ ). Now for $a \in A^{+}$we set $a=a_{1} a_{2}$ where $H=\log a$ and $a_{i}=\exp H_{i}$ as above.

Continuing our integration process described at the end of Section 2 and allowing $F$ to vary we see that the function $\operatorname{Res} f$ is a finite sum of functions of the form

$$
\tilde{\eta}_{\nu}(a)=\tilde{\eta}_{\nu}\left(a_{1}, a_{2}\right)=\sum_{\mu \in L} \eta_{\nu-\mu}\left(a_{1}\right) e^{(i \nu-\rho-\mu)\left(\log a_{2}\right)}
$$

where $\eta_{\nu-\mu}\left(a_{1}\right) \in$ End $\left(V^{M}\right),-H_{\operatorname{Im} \nu} \in \mathfrak{U}^{+}, L$ is the semilattice described in Section 2, the series converges absolutely for $a \in A^{+}$and $\tilde{\eta}_{\nu}(\alpha)=0$ for $\sigma\left(a_{1}\right)>\mathrm{A}$ as do all $\eta_{\nu-\mu}$ 's.

The following lemma is an immediate consequence of this expansion.

LEMMA 4.1. If $\operatorname{Res} f(a)=0$ for all $a \in A^{+}$with $\sigma(a)>C$ then $\operatorname{Res} f=0$. 
THEOREM 4.1. If $G$ has split rank one Res $f$ extends to a (quasi) cusp form. If $G$ has only one conjugacy class of Cartan subgroup Res $f=0$.

Proof. The case where $G$ has split rank one has been treated in [13] and the case where $G$ has only one conjugacy class of Cartan subgroup follows from Lemma 4.1.

COROLlaRY. Suppose $G$ has split rank one or has only one conjugacy class of Cartan subgroup. Then if $f \in C_{c}^{\infty}(G, \tau) f=f_{\varepsilon}$.

\section{§5. Applications to differential equations}

Let $U(\mathbb{S})$ be the complexified enveloping algebra of $\mathbb{S}$ and let $U(\mathbb{S})^{\Omega}$ be the centralizer of $\AA$ in $U(\mathbb{S})$. If $f \in C^{\infty}(G)$ and $X \in \mathbb{S}$ set $X f(g)=$ $\left.(d / d t) f(\exp -t X g)\right|_{t=0}$ and extend this action to all of $U(\mathbb{S})$. Let $\mathscr{E}^{\prime}(G)$ denote the distributions with compact support.

In [14] a sufficient condition for $D \in U(\mathbb{S})^{\Re}$ to be injective as an operator $D: \mathscr{E}^{\prime}(G) \rightarrow \mathscr{E}^{\prime}(G)$ was established. In this section we prove the converse of this result. We first recall the definition of the principal series.

Let $\omega: M \rightarrow G l(H)$ be an irreducible unitary representation of $M$ and let $\nu \in \mathfrak{A}_{\boldsymbol{C}}^{*}$. $\quad \omega$ and $\nu$ define a representation $\nabla_{\omega, \nu}$ of the group $M A N=B$ on $H$ by setting $\nabla_{\omega, \nu}(\operatorname{man})=e^{(i \nu+\rho)(\log a)} \omega(m) \quad(m \in M, a \in A, n \in N)$. Now let $H^{\omega, \nu}$ be the set of all measurable functions $f: G \rightarrow H$ such that:

1) $f(g p)=\nabla_{\omega, \nu}(p)^{-1} f(g)(g \in G, p \in B)$; and,

2) $\int_{K}\|f(k)\|^{2} d k=\|f\|^{2}<\infty$.

Now $H^{\omega, \nu}$ becomes a Hilbert space with inner product

$$
(u, v)=\int_{K}(u(k), v(k)) d k
$$

and left translation induces a representation $\pi_{\omega, \nu}$ of $G$ on $H^{\omega, \nu}$ and we call the pairs $\left(\pi_{\omega, \nu}, H^{\omega, \nu}\right)$ the principal series of $G$. Let $K^{\omega, \nu}$ denote the $K$-finite vectors of $H^{\omega, \nu}$. Observe that $\pi_{\omega, \nu}$ induces a representation of $U(\varangle)$ on $X^{\omega, \nu}$ and that as a $K$-module $X^{\omega, \nu}$ is isomorphic to the space $X(\omega)=\left\{u: K \rightarrow H: u\right.$ is left $K$-finite and $u(k m)=\omega(m)^{-1} u(k)$ for all $k \in K$, $m \in M\}$. We abuse notation and identify $X^{\omega, \nu}$ with $X(\omega)$.

We now restate Lemma 3.1 of [14]. (Injectivity criterion) Suppose 
$D \in U(\mathbb{S})^{\text {R. }}$. Suppose for no $\omega \in \hat{M}$ is there a finite dimensional subspace $U \subseteq X(\omega)$ such that $\pi_{\omega, \nu}(D): U \rightarrow U$ and $\left.\operatorname{det} \pi_{\omega, \nu}(D)\right|_{U}=0$ for all $\nu$. Then $D: \mathscr{E}^{\prime}(G) \rightarrow \mathscr{E}^{\prime}(G)$ is injective.

Observe that $\pi_{\omega, \nu}$ defines a linear map

$$
\pi_{\omega, \nu}: C_{c}^{\infty}(G, \tau) \longrightarrow L\left(H^{\omega, \nu}, V \otimes H^{\omega, \nu}\right)
$$

by setting

$$
\pi_{\omega, \nu}(f) u=\int_{G} f(x) \pi_{\omega, \nu}(x) u d x \quad\left(f \in C_{c}^{\infty}(G, \tau), u \in H^{\omega, \nu}\right) .
$$

If we set $\theta_{\omega, \nu}(f)=\sum_{i=1}\left(\pi_{\omega, \nu}(f) u_{i}, u_{i}\right)$ where $\left\{u_{i}: i \geqq 1\right\}$ is an orthonormal basis of $H_{\omega, \nu}$ we obtain by a simple calculation that $\theta_{\omega,-\nu}\left(\ell(x)^{-1} f\right)=$ $E\left(\psi_{f}(\omega: \nu): \nu: x\right)$ where $\ell(x)(r(x))$ denotes left (right) translation by $x$. (Although the Eisenstein integral may be obtained from a distribution on $G$ our treatment here is useful in the study of differential equations.)

We may now select $u_{1}, \cdots, u_{d}$ an orthonormal set of vectors in $H^{\omega,-\nu}$ such that

$$
\begin{aligned}
\theta_{\omega,-\nu}\left(\ell(x)^{-1} D f\right) & =\theta_{\omega,-\nu}(r(x) D f) \\
& =\sum_{i=1}^{d}\left(\pi_{\omega,-\nu}(D) \pi_{\omega,-\nu}(r(x) f) u_{i}, u_{i}\right)
\end{aligned}
$$

where for $h \in C_{c}^{\infty}(G)$

$$
\left(\pi_{\omega,-\nu}(h) u_{i}, u_{i}\right)=\int_{G} h(x)\left(\pi_{\omega,-\nu}(x) u_{i}, u_{i}\right) d x .
$$

We now prove the converse of the injectivity criterion.

Suppose that $D \in U(\mathbb{S})^{\Re}$ and for $\omega_{0} \in \hat{M}$ we have a finite dimensional $K$-invariant subspace $U \subseteq X\left(\omega_{0}\right)$ such that $\pi_{\omega_{0}, \nu}(D): U \rightarrow U$ and $\left.\operatorname{det} \pi_{\omega_{0}, \nu}(D)\right|_{U}$ $=0$ for all $\nu \in \mathfrak{X}_{C}^{*}$. Without loss of generality we may assume that $\pi_{\omega_{0}, \nu}(D) \equiv 0$ on $U$. Let $\tau$ be the representation of $K$ on $U$ and let $V=$ End $U$ and extend $\tau$ to a double representation of $K$ on $V$.

Now let $F: \hat{M} \times \mathfrak{A}_{c}^{*} \rightarrow V^{M}$ be such that $F(\omega: \nu)=0$ if $\omega \neq s \omega_{0}$ for some $s \in W$. Suppose also that $F$ satisfies conditions I, II and III of Section 3. Set

$$
f(x)=\sum_{\omega \in \hat{M}} \int_{\mathfrak{x}^{*}} E(F(\omega: \nu): \nu: x) \mu(\omega: \nu) d y .
$$

There is an $H \in \mathscr{C}_{q}(G, \tau)$ such that $f+H \in C_{c}^{\infty}(G, \tau)$. Also a simple 
calculation yields

$$
D f(x)=\sum_{\omega \in \hat{M}} \int_{\mathfrak{x}^{*}} E\left(\pi_{\omega,-\nu}(D) \circ F(\omega: \nu): \nu: x\right) u(\omega: \nu) d \nu
$$

and thus $D f=0$ and if $G=f+H$ we see that $D G \in \mathscr{C}_{q}(G, \tau) \cap C_{c}^{\infty}(G, \tau)$ and by [14] $D G=0$. Hence we have proved

THEOREM 5.1. Suppose $D \in U(\varangle)^{\Re} . \quad D: \mathscr{E}^{\prime}(G) \rightarrow \mathscr{E}^{\prime}(G)$ is injective if and only if for no $\omega \in \hat{M}$ is there a finite dimensional subspace $U \subset X(\omega)$ such that $\pi_{\omega, \nu}(D): U \rightarrow U$ and $\left.\operatorname{det} \pi_{\omega, \nu}(D)\right|_{U}=0$ for all $\nu \in \mathfrak{Y}_{c}^{*}$.

For $r>0$ let $V_{r}(0)=\{g \in G: \sigma(g) \leq r\}$

THEOREM 5.2 (P-convexity). Suppose $D \in U(\mathbb{S})^{\mathfrak{s}}$ satisfies the injectivity criterion. Suppose $T \in \mathscr{E}^{\prime}(G)$ and $\operatorname{supp} D T \subseteq V_{r}(0)$. Then $\operatorname{supp} T \subseteq V_{r}(0)$.

Proof. By convoluting with functions in $C_{c}^{\infty}(G)$, we see that it suffices to prove this result for $T=f \in C_{c}^{\infty}(G)$. Furthermore, it suffices to assume that $f(x)=L(F(x))$ where $F \in C_{c}^{\infty}(G, \tau), V=$ End $U, U$ is a $K$ finite space of functions on $K, L \in V^{*}$ and $\tau$ is the double representation induced on $V$ by left translation on $U$.

By hypothesis for all $N>0$ there is a $C_{N}$ such that

$$
\left|\psi_{D F}(\omega: \nu)\right| \leq C_{N}(1+\|\nu\|)^{-N} e^{r\|\operatorname{Im} \nu\|}
$$

but as $\psi_{D F}(\omega: \nu)=\pi_{\omega,-\nu}(D) \psi_{F}(\omega: \nu)$ we have that $\psi_{F}(\omega: \nu)$ satisfies the same growth conditions. Thus, as $F \in C_{c}^{\infty}(G, \tau)$ we have $\operatorname{supp} F \subseteq V_{r}(0)$ and hence supp $f \subseteq V_{r}(0)$.

\section{REFERENCES}

[1] J. Arthur, Harmonic analysis of the Schwartz space on a reductive Lie group II, preprint.

[2] L. Ehrenpreis and F. Mautner, Some properties of the Fourier transform on semisimple Lie groups, I, Ann. of Math. 61 (1955), 406-439.

[ 3 ] R. Gangolli, On the Plancherel formula and the Paley-Wiener theorem for spherical functions on semisimple Lie groups, Ann. of Math. 93 (1971), 150-165.

[4] S. G. Gindikin and F. I. Karpelevic, Plancherel measure of Riemannian symmetric spaces of nonpositive curvature, Sov. Math. 3 (1962), 962-965.

[ 5 ] Harish-Chandra, Discrete series for semisimple Lie groups II, Acta. Math. 116 (1966), 1-111.

[ 6 ] — On the theory of the Eisenstein integral, Proc. Int. Conf. on Harm. Anal., Univ. of Maryland, 1971, lecture notes in Math. No. 266, Springer-Verlag, 1972.

[ 7 ] - Lectures at Institute for Advanced Study, Fall 1974. 
[ 8 ] S. Helgason, An analog of the Paley-Wiener theorem for the Fourier transform on certain symmetric spaces, Math. Ann. 165 (1966), 297-308.

[9] - A duality for symmetric spaces, with applications to group representations, Advan. Math. 5 (1970), 1-154.

[10] - The surjectivity of invariant differential operators on symmetric spaces I, Ann. of Math. 98 (1973), 451-479.

[11] L. Hormander, Linear partial differential operators, Springer-Verlag, 1963.

[12] K. Johnson, Functional analysis on SU (1,1), Advan. Math. 14 (1974), 346-364.

[13] — Paley-Wiener theorems on groups of split rank one, to appear.

[14] - Partial differential equations on semisimple Lie groups, to appear.

[15] G. Schiffmann, Integrales d'entrelacement et fonctions de Whittaker, Bull. Soc. Math. France 99 (1971), 3-72.

[16] G. Warner, Harmonic analysis on semisimple Lie groups, Springer-Verlag, 1972.

Department of Mathematics

Indiana University 